\title{
Tandem Mirror Experiment for Basic Fusion Science
}

\author{
Wendell Horton', Igor Alvarado², Xiangrong Fư ${ }^{3}$, Alexei Bekemishev ${ }^{4}$ \\ ${ }^{1}$ Institute for Fusion Studies, The University of Texas at Austin, Austin, USA \\ ${ }^{2}$ National Instruments Corporation, Austin, USA \\ ${ }^{3}$ Los Alamos National Laboratory, Los Alamos, USA \\ ${ }^{4}$ Budker Institute of Nuclear Physics, Siberian Branch of the Russian Academy of Sciences, Novosibirsk, Russia \\ Email: wendell.horton@gmail.com
}

Received 27 November 2013; revised 3 January 2014; accepted 15 January 2014

Copyright (C) 2014 by authors and Scientific Research Publishing Inc.

This work is licensed under the Creative Commons Attribution International License (CC BY).

http://creativecommons.org/licenses/by/4.0/

(c) (i) Open Access

\section{Abstract}

Research on controlled nuclear fusion has been largely concentrated on plasma confinement using toroidal magnetic fields. Toroidal systems are complex. A simpler magnetic confinement system may provide a valuable platform for understanding fusion plasmas. The linear mirror machine has delivered good performance with the potential of giving a direct conversion of nuclear energy into electric power. The GAMMA-10 (G-10) linear mirror confinement system at Tsukuba University demonstrated the principle of the direct conversion of plasma energy into electric power on a small scale from the exhaust plasma in the exterior divertor chamber. The tokamak fusion system has to prove that the 10 to 15 MA of plasma current can be sustained continuously with acceptable efficiency. Plasma confinement is due to the magnetic field from the plasma current in tokamaks. There is room for creative new solutions in the magnetic confinement of fusion plasmas, and consideration is given for the alternative approach of using a linear machine with high magnetic mirror fields and the direct conversion of the high temperature escaping plasma to electric power.

\section{Keywords}

Controlled Nuclear Fusion, Plasma Confinement, Toroidal Magnetic Fields, GAMMA-10, Gas Dynamic Trap

\section{Introduction to Tandem Mirror Plasma Science}

The technologies (diagnostics, real-time controls, material radiation limits) are simpler in the open mirror magnetic confinement system. The linear confinement system has lower technological challenges. For example, the 
Earth's magnetosphere is a mirror confinement system that traps relativistic electrons for months. Our fundamental understanding of plasma confinement in the tandem mirror system is rudimentary owing to the lack of funding for the mirror research since the 1980s. At that time the US-Department of Energy support for mirror research program was terminated with the understanding that the tokamak would produce fusion power within 20 to 30 years. Japan and Russia continued to pursue a vigorous mirror research program with the construction of the G-10 device in Tsukuba, Japan and the Gas Dynamic Trap (GDT) in Novosibirsk, Russia. Important advances have been made in the past ten years from these two large, magnetic fusion plasma confinement devices.

New mirror physics confinement results have important implication for the plasma confinement time scaling laws and for the scaling laws for neutron sources. These plasma science results are needed to make a decision on how to design a practical fusion reactor or a hybrid fusion-fission power system. A new mirror plasma confinement experiment would give important results both for $14 \mathrm{MeV}$ neutron sources plasma-wall interaction and for the hybrid fusion-fission power reactor design. The linear mirror confinement machine is relatively simple and easily built compared with toroidal systems. There is agreement that the time is right to start a university-industry collaborative program based on an Axisymmetric Tandem Mirror (ATM).

The program would achieve the goals advocated by a leading mirror machine scientist, Dr. Dmitry Ryutov, of the Lawrence Livermore National Laboratory in California, of providing a high-powered neutron source in the near future [1].

Experimental research on alternative concepts, such as the ATM, would be fundamental in improving understanding of the plasma magnetic confinement. As stated in a recent Department of Energy Congressional Budget request (FY 2010, Science, Vol. 4, Fusion Energy Sciences), "the smaller scale experiments and the cuttingedge research have proven to be effective in attracting students and strongly contribute to fusion workforce development and the intellectual base of the fusion program". In the same document it is stated that "research on alternate concepts is pursued to develop a fuller understanding of the physics of magnetically confined plasma..." "Ongoing theory and modeling efforts, aimed at developing a predictive capability for the operation of fusion experiments, will continue as will enabling technology development".

Described here is the first step along a new path with simpler linear geometry that could make a significant contribution for a better understanding and predictive tools for plasma science. The ATM system has lowtransverse ion transport-owing to the azimuthal symmetry which gives simpler and better-confined particle orbits. It has less stability for the plasma eigenmodes, but several efficient ways for ensuring stability of axisymmetric systems or operating with persistent modes have been found [2]. Scientists in the Mirror Forum Group, formed in the fall of 2008, argued that the tandem mirror would lead to a simpler, high-powered neutron source in the near future at modest cost. The system would have real-time control of the mirror-confined plasma [3]. Field-programmable gate-arrays (FPGA) with other advanced hardware/software systems including Graphics Processing Units (GPUs) would be incorporated into the diagnostic suite for near real-time input-output [RIO] control in the steady state mirror magnetic fusion confinement plasma system (http://www.ni.com).

The key plasma diagnostics for the mirror plasma are Thomson scatterings and tomographic reconstruction $\mathrm{X}$-rays images of the plasma dynamics. Thomson scattering system gives accurate measurements of the electron temperature profiles. Despite the high-ion temperatures achieved, the fusion power relevance of the Japanese and Russian mirror confined plasma is clouded by the lack of this "gold standard" method for determining the electron temperature profiles. For example, earlier the G-10 device reported the core electron temperature of 800 $\mathrm{eV}$, which, if verified, would open the door to designing the next fusion neutron sources [4] [5]. Recently, however, Yoshikawa et al. [6] report that new Thomson scattering experiment finds the core electron temperature at $80-100 \mathrm{eV}$ in G-10. It is not clear if the two plasmas separated by 10 years are the same, but the divergence emphasizes the need for validated methods of measuring the electron temperatures. At a Re New Workshop organized by the Office of Fusion Energy Science in 2005, the panel suggested that the $800 \mathrm{eV}$ measurement was not a true temperature but rather a high-energy component connected to a lower-temperature thermal (Maxwell-Boltzmann) electron distribution function. The soft X-ray spectrum measurements used by the G-10 group could not answer this question. This issue is a classic problem in laboratory plasma physics. A similar doubt was raised about the initial results of the tokamak $T_{e}(r)$-data reported by Artsimovich in 1967 at the Princeton Plasma Physics Laboratory (PPPL). The issue in the tokamak was resolved using a Thomson scattering measurement with lasers invented only a few years earlier at UC Berkeley-showing the importance of international collaboration.

A new phase of the Thomson scattering and X-ray diagnostic systems with the real-time analysis and control 
systems to be built into the magnetic mirror facilities is briefly described, with expansion on the Thomson laser scattering diagnostic designed by LeBlanc [7] on NSTX at PPPL. The plan is to use 10 laser beams through the radius of the cylindrical plasma of the tandem mirror machine with longer laser pulses than used in the NSTX device for collecting sufficient electron scattered photons. Signal processing would be performed in real time using state-of-the-art instrumentation and computations made possible by PIXI data acquisition system and the Compact RIO using FPGAs. Graphics Processing Units (GPUs) would also be used for high speed imagining of the plasma. This would allow real time solutions of MHD background plasma with the concomitant drift-wave turbulence that drives the anomalous radial transport. Real-time commands would be issued to the external neutral beam and radio frequency (RF) plasma heating systems to bring the plasma back to the target state.

\section{Attractive Features of Mirror Machines for Near-Term Steps in Plasma Science}

The mirror device was recognized at the beginning of the search for fusion power by the US, France, England and Russia as an attractive method for confining hot plasmas. The mirror program centered at the Lawrence Livermore National Laboratory was terminated in the US in the 1980s. All resources have currently flowed into the tokamak approach. Tokamaks have achieved the highest triple product measure of density times confinement times plasma temperatures. The progress in tokamaks that was rapid in the 1980s and 1990s has slowed appreciably since the 1990s and has not led to the demonstration of ignition, nor to net fusion power amplification (fusion $Q>1$ ). JET (Joint European Torus) achieved the maximum ratio of fusion power to injected power for a few seconds in 1994 with this ratio $Q=17 \mathrm{MW} / 26 \mathrm{MW} \approx 2 / 3$. Thus, the time may be right to again look more carefully at the mirror plasma confinement device. More than just another fusion effort, it is believed that a new laboratory experiment with an ATM system would have a broad impact in plasma science discovery and understanding. This new understanding from laboratory mirror confinement experiments would be useful to space physics, astrophysics, material plasma-surface interaction physics and plasma processing systems.

The ATM system offers several important advantages to the complex toroidal confinement systems. The GDT, shown in Figure 1, also has large expansion tanks and obtains record-high electron temperatures of $430 \mathrm{eV}$ by Thomson scattering [8]. Advantages following the simple, linear geometry include 1) that single electrons, ions and alpha particles are confined by a mathematical theorem (KAM) theory for the axisymmetric geometry and 2) that there is no neoclassical transport as in tokamaks due to absence of banana orbits. Tokamaks confine the particles with the magnetic field produced by the toroidal plasma current rather than an externally generated magnetic field. Controlling the required 10 - 15 MA toroidal plasma current is a challenge requiring high-power $\mathrm{GHz}$ RF waves injected from complex antennas on the chamber walls to transfer wave momentum to the plasma electrons [9]. A list of advantages for the mirror system is as follows:

1) The mirror plasma has a natural magnetic divertor that sends the radially-escaping plasma particles with

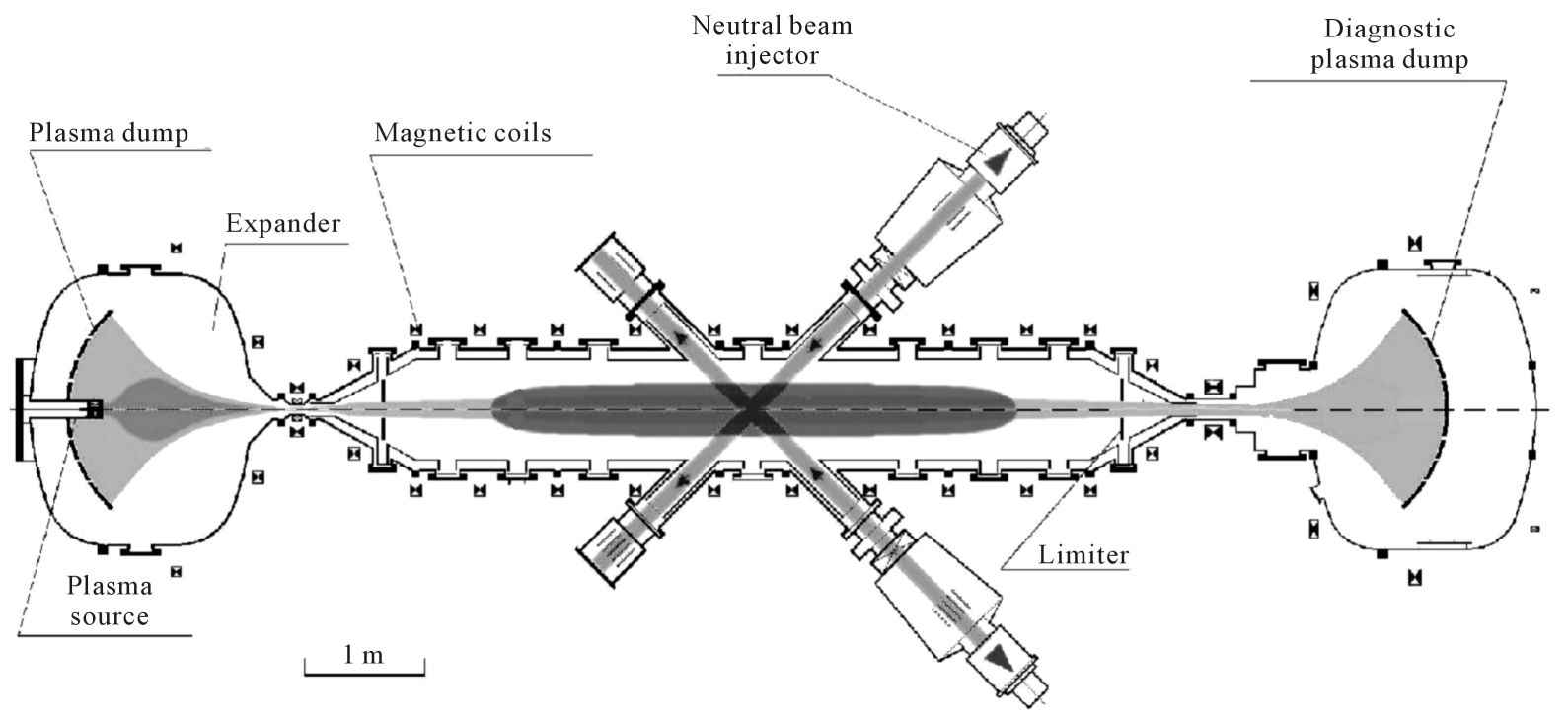

Figure 1. Gas dynamic trap in Novosibirsk at the Budker Institute. 
their high kinetic energy out the ends through an expanding magnetic nozzle as shown in Figure 1. The end nozzles means the divertor is external to the plasma confinement region in contrast to tokamaks where the divertor is part of the confinement vessel. The external divertor allows the introduction of a direct electric power conversion from the escaping plasma. The promise of a direct conversion from plasma energy into electricity-thus avoiding the use the neutron flux thermal exchange to drive a mechanical-electric generator has considerable advantages. The efficiency of the steam driven mechanical-electric generator is below $50 \%$.

2) The mirror plasma is a neutron source with advantages as described by Ryutov et al. [1], Post et al. [10] [11] and Ivanov [12]. The mirror group at the Budker Institute is currently designing a mirror machine to produce a $14 \mathrm{MeV}$ neutron source for the determining the life-time of wall materials exposed to the neutron and plasma fluxes [13].

3) Kulsrud et al. [14] [15] show that by using nuclear spin-polarized deuterons and tritons in a straight mirror central cell there is an enhanced production of neutrons. This polarization mechanism works in the straight magnetic field lines in the central cell and is not feasible in strongly-varying toroidal magnetic fields.

4) The linear geometry device is simpler to build and maintain in the radioactive environment. The architecture of the diagnostics is simpler in the linear cylindrical geometry.

Mirror traps have excellent single-particle confinement in contrast to both tokamak and helical/stellarator magnetic traps. In the case of tokamaks, there is no confinement from the vacuum-magnetic fields and in helical/ stellarators toroidal systems there are many escaping chaotic particle orbits in the helical magnetic field. For example, in ITER, confinement of the alpha particles requires the full $15 \mathrm{MA}$ of plasma current. Even so, the radial excursions of a confined $3.5 \mathrm{MeV}$ alpha particles will be $30 \mathrm{~cm}$-more than $10 \%$ of the plasma radius. Other alpha particles are promptly lost and may damage the walls of the toroidal chamber walls. Thus, the role of a new, well-diagnosed mirror plasma experiment with the real-time controls, for example, with PXI and Compact RIO systems, has the potential of advancing fusion science and building a broader engineering and science base for the future magnetic fusion plasma reactors.

There are some problems associated with the mirror-confined plasmas. Up till the recent results from the GDT and G-10, the mirror devices have been plagued with low-electron temperatures-less than $100 \mathrm{eV}$. The GAMMA-10 machine shown in Figure 2 at Tsukuba University published data indicating electron temperatures of order $500 \mathrm{eV}$, and then subsequently reported a maximum of $800 \mathrm{eV}$ [4]. The new report of Yoshikawa et al. [6] gives a lower temperature for a discharge that may be different from those in the early 2000 experiments. The proposed research would investigate this divergence with an international group of scientists and engineers.

The reason that a portion of the electron distribution is expected to be high energy in the G-10 device is that there are large expansion tanks at the ends of the G-10 machine that provide thermal isolation of the hot trapped electrons from the metallic end surfaces.

The simplicity of the axisymmetric tandem geometry in a linear cylindrical configuration is highly desirable.

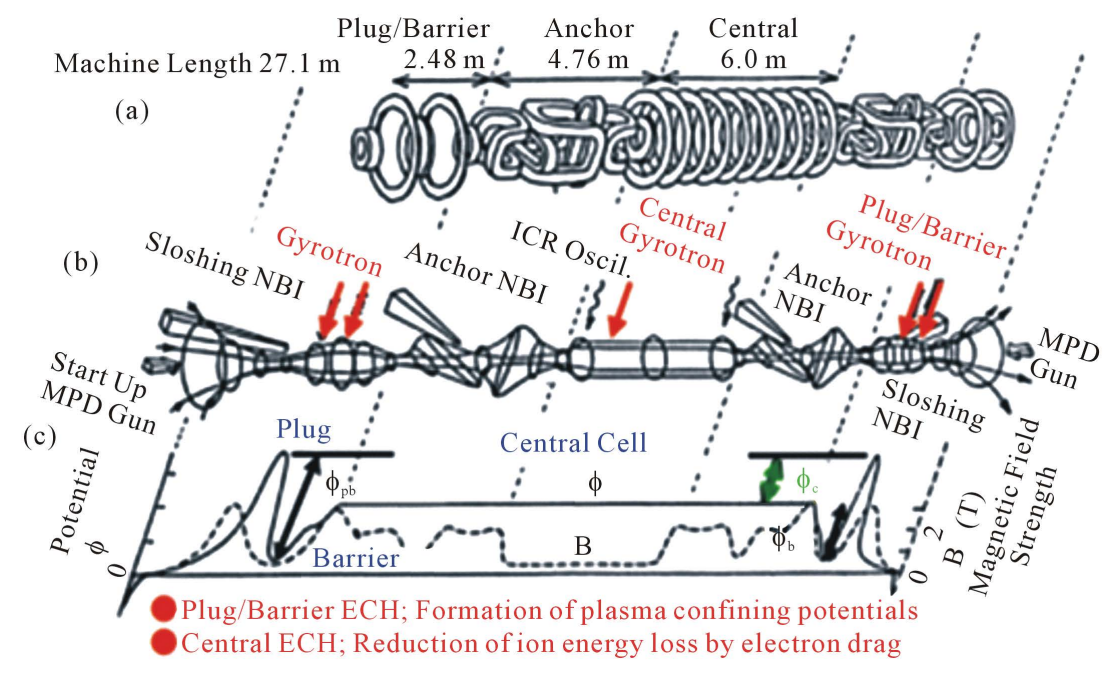

Figure 2. Gamma-10 magnet and power systems are shown with a typical axial potential profile. 
The mirror plasma is a high-plasma energy density system. In the Earth's magnetosphere, for example, stable high-energy density plasmas are known to be confined by the planetary magnetic field through the mirror confinement principle. There are long-lived $\mathrm{MeV}$ electrons and $500 \mathrm{KeV}$ protons confined in the inner magnetosphere. The technical reason for the enhanced stability of these devices compared with similar sized toroidal plasma is the absence of the destabilizing magnetic curvature that covers the outer half volume of the torus. In the magnetic mirror there are locally unstable regions but they are adjacent to and strongly connected to stable regions. The result is that the net stability is much stronger up to high plasma energy densities in the mirror system [16].

The toroidal geometry exacerbates the level of turbulence that produces the fast losses of plasma energy across the magnetic field. This physics results in the confinement time decreasing as the heating power and plasma temperature increase in the toroidal system [17]. In the high-beta mirror system this effect is weaker and may be subdominant, as shown by Pratt and Horton [18] and Berk and Pratt [19]. In sharp contrast, the mirror confinement improves with higher heating power in the experiments reported from University of Tsukuba in Japan and the Budker Institute for Nuclear Physics in Russia.

\section{Conclusions}

In conclusion, there are distinct advantages of the symmetric mirror plasma confinement system, whereas, the toroidal plasmas are limited to plasma pressures of less than $5 \%-8 \%$ of the magnetic pressure, owing to the large uniform, unfavorable magnetic curvature over the entire outer half of the toroidal surface. Mirror-confined plasmas are stable at plasma pressures close to the magnetic field pressure. This magnetic curvature results in the magnetic version of the Rayleigh-Taylor instability on all space scales from the size of the machines radius down to the scale of the electron gyroradius.

The small-scale turbulence is the mechanism responsible for the large cross-field electron thermal diffusivity that prevents the electron temperature from increasing in proportion to the auxiliary heating power. Plasma data shows only a slow increase of electron temperature with auxiliary heating power due to the increasing strength of the small (millimeter) scale plasma turbulence that rises in intensity with increased heating power.

The mirror plasma has a natural divertor that sends the escaping plasma particles and energy out through a nozzle along the ends of the cylindrical axis into an expansion chamber. This end nozzle has allowed the Kobe University research team to build a cusp module that fits on the ends of the G-10 machine that produces a direct conversion of escaping plasma power to electric power. The promise of a direct conversion from plasma energy into electricity thus avoiding use of the heat from neutrons to power a steam driven electric generator has great advantages.

\section{Acknowledgements}

The research is supported by the Department of Energy-Magnetic Fusion Energy, through a grant to the Institute for Fusion Studies and by the National Science Foundation Grant 0964692 to The University of Texas at Austin, Austin, Texas, Los Alamos Scientific Laboratory, Los Alamos, New Mexico and the Budker Institute of Nuclear Physics, Novosibirsk, Russia. The authors thank Mrs. Suzy Mitchell for her typing and formatting skills for this project. Mrs. Mitchell worked from 1981-2002 as a Senior Technical Secretary. In 2002, she retired but continues to this present day doing freelance work from the Hill Country of Texas.

\section{References}

[1] Ryutov, D.D., Baldwin, D.E., Hooper, E.B. and Thomassen, K.I. (1998) A High-Flux Source of Fusion Neutrons for Material and Component Testing. Journal of Fusion Energy, 17, 253-257. http://www.springerlink.com/openurl.asp?id=doi:10.1023/A:1021870631838 http://dx.doi.org/10.1023/A:1021870631838

[2] Ryutov, D.D., Berk, H.L., Cohen, B.I., Molvik, A.W. and Simonen, T.C. (2011) Magneto-Hydrodynamically Stable Axisymmetric Mirrors. Physics of Plasmas, 18, Article ID: 092301. http://dx.doi.org/10.1063/1.3624763

[3] Giannone, L., Wenzel, L., et al. (2009) Data Acquisition and Real Time Signal Processing of Plasma Diagnostics on ASDEX Upgrade Using LabVIEW RT. International Atomic Energy Agency, Wien.

[4] Cho, T., et al. (2006) Progress in Potential Formation and Radial-Transport-Barrier Production for Turbulence Suppression and Improved Confinement in GAMMA-10. Annual Report National Institute for Fusion Science. University 
of Tsukuba, Tsukuba.

[5] Cho, T., et al. (2008) Active Control of Internal Transport Barrier Formation Due to Off-Axis Electron-Cyclotron Heating in GAMMA-10 Experiments. Physics of Plasmas, 15, Article ID: 056120. http://link.aip.org/link/?PHPAEN/15/056120/1 http://dx.doi.org/10.1063/1.2906262

[6] Yoshikawa, M., Nagasu, K., Shimamura, Y., Shima, Y., Kohagura, J., Kitagawa, K., Morishita, M., Sakamoto, M., Nakashima, Y., Imai, T., Ichimura, M., Kawarazaki, R., Yamada, I., Yasuhara, R., Funaba, H. and Minami, T. (2006) Electron Temperature and Density Measurements by Using the Thomson Scattering System in the Tandem Mirror GAMMA-10. 16th International Conference on Laser-Aided Plasma Diagnostics (26th LAPD), Madison, 22-26 September 2013. http://link.aps.org/doi/10.1103/PhysRevLett.97.055001

[7] LeBlanc, B.P., Bell, R.E., Johnson, D.W., et al. (2003) Operation of the NSTX Thomson Scattering system. Review of Scientific Instruments, 74, 1659-1662. http://dx.doi.org/10.1063/1.1532763

[8] Solomahin, A., Bagryansky, P., et al. (2014) Enigorod International Conference Plasma Physics and Controlled Fusion. Zvenigorod, Russia.

[9] Horton, W., Goniche, M., Peysson, Y., Decker, J., Ekedahl, A. and Litaudo, X. (2013) Penetration of Lower Hybrid Current Drive Waves in Tokamaks. Physics of Plasmas, 20, Article ID: 112508. http://dx.doi.org/10.1063/1.4831981

[10] Post, R.F. (2002) The Kinetic Stabilizer: Further Calculations and Options, Transactions of Fusion Science and Technology, American Nuclear Society. The 4th International Conference on Open Magnetic Systems for Plasma Confinement, Jeju Island, 1-4 July 2002, 195-202.

[11] Post, R.F., Fowler, T.K., Killeen, J. and Mirin, A.A. (1973) Concept for a High-Power-Density Mirror Fusion Reactor. Physical Review Letters, 31, 280-282. http://link.aps.org/doi/10.1103/PhysRevLett.31.280 http://dx.doi.org/10.1103/PhysRevLett.31.280

[12] Ivanov, A.A., Beklemishev, A.D., Kruglyakov, E.P., Bagryansky, P.A., Lizunov, A.A., Maximov, V.V., Murakhtin, S.V. and Prikhodko, V.V. (2010) Results of Recent Experiments on GDT Device after Upgrade of Heating Neutral Beams. Fusion Science and Technology, 57, 320-325.

[13] Molvik, A.W., Ivanov, A.A., Kulcinski, D., Ryutov, D.D., Santarius, J., Simonen, T., Wirth, B.D. and Ying, A. (2010) A Gas Dynamic Trap Neutron Source for Fusion Material and Subcomponent Testing. Fusion Science and Technology, 57, 369-394.

[14] Kulsrud, R.M., Valeo, E.J. and Cowley, S.C. (1986) Physics of Spin-Polarized Plasmas. Nuclear Fusion, $26,1443$.

[15] Kulsrud, R.M., Furth, H.P., Valeo, E.J. and Goldhaber, M. (1982) Fusion Reactor Plasmas with Polarized Nuclei. Physical Review Letters, 49, 1248-1251. http://link.aps.org/doi/10.1103/PhysRevLett.49.1248 http://dx.doi.org/10.1103/PhysRevLett.49.1248

[16] Crabtree, C., Horton, W., Wong, H.V. andVan Dam, J.W. (2003) Bounce-Averaged Stability of Compressional Modes in Geotail Flux Tubes. Journal of Geophysical Research, 108, 1084. http://www.agu.org/pubs/crossref/2003/2002JA009555.shtml http://dx.doi.org/10.1029/2002JA009555

[17] Horton, W. (2012) Turbulent Transport in Magnetized Plasmas, Chapters 12-15. World Scientific, Singapore City. http://dx.doi.org/10.1142/8362

[18] Pratt, J. and Horton, W. (2006) Global Energy Confinement Scaling Predictions for the Kinetically Stabilized Tandem Mirror. Physics of Plasmas, 13, Article ID: 042513. http://link.aip.org/link/?PHPAEN/13/042513/1 http://dx.doi.org/10.1063/1.2188913

[19] Berk, H.L. and Pratt, J. (2011) Trapped Particle Stability for the Kinetic Stabilizer. Nuclear Fusion, 51, Article ID: 083025. http://iopscience.iop.org/0029-5515/51/8/083025 http://dx.doi.org/10.1063/1.2188913 